\title{
Modeling of construction process scheduling with Time Coupling Methods
}

\author{
Zdzisław Hejducki and Łukasz Łodożyński* \\ Wrocław University of Science and Technology, Poland
}

\begin{abstract}
The topic of the paper is referred to optimization model for multiobjective solutions for construction management. It is known in literature as Time Coupling Methods (TCM). It presents another approach to scheduling methods from known methods like LSM, CPM/PERT, LOC, RSM. TCM is based on couplings that bounds activities in technology and management. The new modifications to TCM will be presented that add flexibility when dealing with complex civil engineering problems.
\end{abstract}

\section{Introduction}

In the planning of construction works there are used, among others: network planning (CPM/PERT), Gantt charts, circular charts (LOB - Line of Balance), linear scheduling (LSM - Linear Scheduling Model, RSM - Repetitive Scheduling Method), Critical Chain Method [4], time couplings methods (TCM) [1,8], and many other methods. The aim of the article is to present a modification of MSC III (one scheduling methods). I adds more flexibility when dealing with technological and organization constrains. The principles of basic method will be provided and then modified by additional constrains.

\section{Basic assumptions}

The Time Coupling Method (TCM III) [4] is one of the methods of construction works scheduling taking into account the simultaneous relationship between available site of works and construction processes. Simultaneous incorporation of technological and organizational constraints enables setting a minimum deadline for works completion. The use of coupling between the means and execution processes allows us to determine the critical work string, which defines a total time limit for completion of the complex construction works. Non-critical works have time buffers characterized by the earliest and latest dates. The calculations are carried out using the matrix model [11], in the columns there are numerical data regarding the execution time of construction processes, while the lines indicate the division into sites of works.

Firstly, the earliest dates of works are calculated. The first column introduces limitations in the form of zero couplings, which ensures continuity of the first type of works. The first row takes into account zero couplings between sites of works, forcing the continuity of

\footnotetext{
*Corresponding author: lukasz.lodozynski@pwr.edu.pl
} 
work on the building site. In the remaining rows and columns, the early dates are determined in accordance with Figure 1. The work cannot start earlier than both: the previous work on this site (technological condition) and work of the same type on the previous site (organizational condition) have been completed. In the second stage, the latest work deadlines should be set. This time, the last column assumes the continuity of the construction process, whereas in the last line, there is continuity of work on the work site assumed. In the remaining rows and columns, the latest dates are determined in accordance with Fig. 1. The work cannot be finished later than the following work starts on this site (technological condition), as well as the work of the same type on the following site (organizational condition). After all the times have been determined, there are, among others, such characteristics available as: temporal, technological, organizational reserves and critical path. In the process of scheduling construction it is proposed to use diagrams.

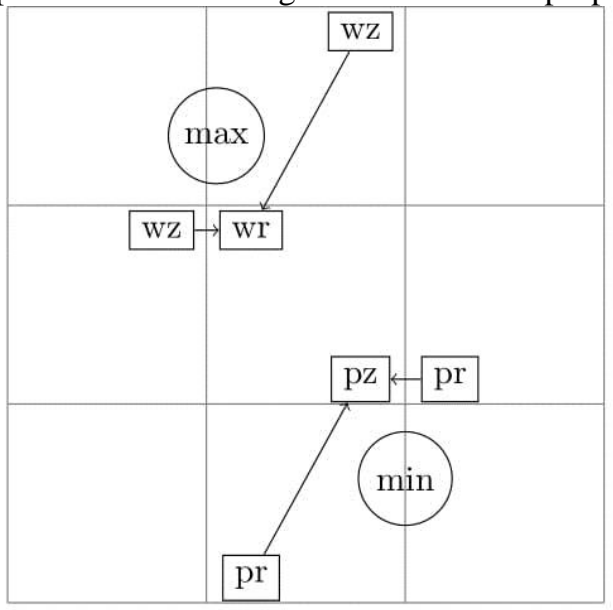

Figure 1: Outline of works dates designation

- $\quad w r$ - the earliest date of starting work,

- $\mathrm{pr}$ - the latest date of starting work,

- $\quad w z-$ the earliest date of completion of work

- $\quad \mathrm{pz}$ - the latest date of completion of work.

\section{General computation model}

The solution is to determine such quantities as: the earliest and latest date of starting and finishing work, couplings in earliest and latest times, time buffers, critical path and total time of project.

$$
T=\sum_{q=1}^{m+n-1} t_{i, j_{q}}
$$

The earliest times of all activities in the first sector $(\mathrm{i}=1)$ are determined by equations ( 2 ) and (3).

$$
t_{1,1}^{w r}=0, \quad t_{1, j}^{w r}=t_{1, j-1}^{w z}
$$




$$
t_{1, j}^{w r}=\sum_{k=1}^{j-1} t_{1, k}, \quad j=\{1,2, \ldots, m-1\}
$$

The earliest times of all sectors in the first activity $(j=1)$ are determined by equations (4) and (5).

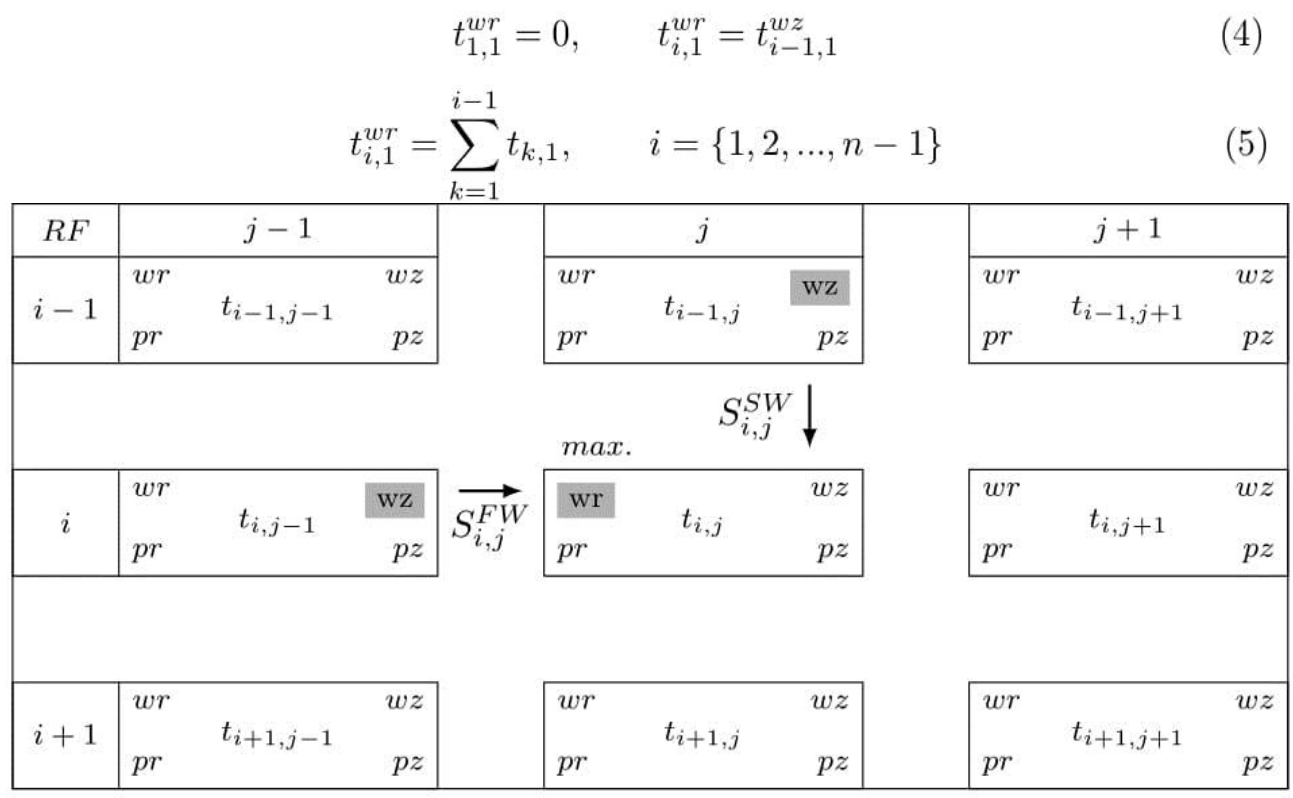

Figure 2: Earliest dates calculation diagram

The couplings for the first activity are zero (as in TCM I) and don't affect the first sector. The couplings for the first sector are zero (as in TCM II) and don't affect the first activity.

$$
\begin{aligned}
& S_{i, 1}^{S W}=0 \\
& S_{1, j}^{F W}=0
\end{aligned}
$$

The earliest times of starting and finishing the work are determined by equations (8) and (9), (Fig. 2\}).

$$
t_{i, j}^{w r}=\max \left\{t_{i-1, j}^{w z}, t_{i, j-1}^{w z}\right\}
$$

The earliest times of finishing the work $(i, j)$ is determined by adding the earliest times of the starting work and it's duration.

$$
t_{i, j}^{w z}=t_{i, j}^{w r}+t_{i, j}
$$

In order to determinate other earliest times of starting the work $(i, j)$ the following couplings needs to be considered:

- The preceding work in activity $(\mathrm{i}-1, \mathrm{j})$ - the continuity of activities

- The preceding work in sector $(\mathrm{i}, \mathrm{j}-1)$ - the continuity of work in sectors

The couplings between activities for the earliest times are determined by difference between earliest times of starting and finishing of two adjacent works in one activity.

$$
S_{i, j}^{S W}=t_{i, j}^{w r}-t_{i-1, j}^{w z}
$$


The couplings between sectors for the earliest times are determined by difference between earliest times of starting and finishing of two adjacent works in one sector.

$$
S_{i, j}^{F W}=t_{i, j}^{w r}-t_{i, j-1}^{w z}
$$

The latest times are determined by an assumption that the earliest and latest times of finishing work in the latest activity in latest sector are equal.

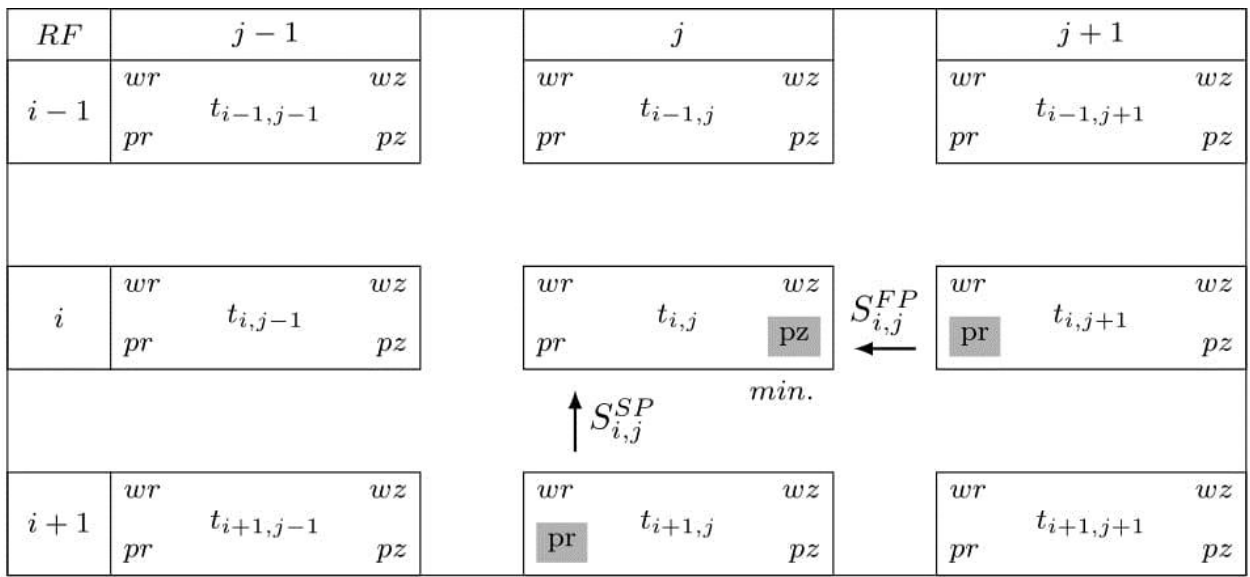

Figure 3: Latest dates calculation diagram

$$
t_{n, m}^{p z}=t_{n, m}^{w z}
$$

The latest times of all activities on last sector ( $\mathrm{i}=\mathrm{n})$ are determined by equations (13) and (14).

$$
\begin{gathered}
t_{n, j}^{p z}=t_{n, m}^{p z}-\sum_{n=j+1}^{p z} t_{n, k}^{p z}, \quad j=\{1,2, \ldots, m-1\} \\
\text { pr } t_{n, j+1}
\end{gathered}
$$

The latest times of all sectors in the last activity $(\mathrm{j}=\mathrm{m})$ are determined by equations (15) and (16).

$$
\begin{gathered}
t_{i, m}^{p z}=t_{i+1, m}^{p r} \\
t_{i, m}^{p z}=t_{n, m}^{p z}-\sum_{k=i+1}^{n} t_{k, m}, \quad i=\{1,2, \ldots, m-1\}
\end{gathered}
$$

Couplings for the last activity are zero (as in TCM I) and don't affect the last sector.

Couplings for the last sector are zero (as in TCM II) and don't affect the last activity.

$$
\begin{aligned}
& S_{i, m}^{S P}=0 \\
& S_{n, j}^{F P}=0
\end{aligned}
$$

The latest times of finishing and starting the work are determined by equations (19) and (20), (Fig. 3).

$$
t_{i, j}^{p z}=\max \left\{t_{i-1, j}^{p r}, t_{i, j-1}^{p r}\right\}
$$

The latest times of starting the work (i,j) is determined by subtracting the latest times of the finishing work and it's duration.

$$
t_{i, j}^{p r}=t_{i, j}^{p z}-t_{i, j}
$$

In order to determinate other latest times of finishing the work $(i, j)$ the following couplings needs to be considered: 
- The following work in activity $(i+1, j)$ - the continuity of activities

- The following work in sector $(i, j+1)$ - the continuity of work in sectors

The couplings between activities for the latest times are determined by difference between latest times of starting and finishing of two adjacent works in one activity.

$$
S_{i, j}^{S P}=t_{i, j}^{p r}-t_{i-1, j}^{p z}
$$

The couplings between sectors for the latest times are determined by difference between latest times of starting and finishing of two adjacent works in one sector.

$$
S_{i, j}^{F P}=t_{i, j}^{p r}-t_{i, j-1}^{p z}
$$

The time reserves for each work are determinate as following: The time reserves are equal to the following values:

$$
R_{i, j}=t_{i, j}^{p r}-t_{i, j}^{w r}=t_{i, j}^{p z}-t_{i, j}^{w z}=t_{i, j}^{p z}-t_{i, j}^{w r}-t_{i, j}
$$

- difference between the latest and earliest time of starting of the work

- difference between the latest and earliest time of finishing of the work

- difference between the latest time of finishing and the earliest time of starting of the work and its duration

\section{Modified model}

In basic method the time between the works in one activity is calculated by the method rules. The additional matrix containing organizational couplings is added. It modifies the couplings in the original method by the couplings provides by the $S^{S}$ matrix. When the $S_{i, j}^{S}>0$ it forces lag between the works in activity. This is the case when workforce needs to rearm, move, rest, etc. When $S_{i, j}^{S}<0$ it forces less (possibly no) lags between the works in activities. If the lag is zero then it ensures continuity of selected works in activity (e.g. seamless concreting).

$$
\mathbf{S}^{\mathbf{S}}=\left[\begin{array}{ccccc}
S_{1,1}^{S} & S_{1,2}^{S} & \ldots & \ldots & S_{1, m}^{S} \\
S_{2,1}^{S} & S_{2,2}^{S} & \ldots & \ldots & S_{2, m}^{S} \\
\vdots & \vdots & S_{i, j}^{S} & \ldots & \vdots \\
S_{n-1,1}^{S} & \ldots & \ldots & \ddots & S_{n-1, m}^{S}
\end{array}\right]
$$

The earliest times of all activities in the first sector (i=1) are determined by equations (24) and (25).

$$
\begin{gathered}
t_{1,1}^{w r}=0, \quad t_{1, j}^{w r}=t_{1, j-1}^{w z} \\
t_{1, j}^{w r}=\sum_{k=1}^{j-1} t_{1, k}, \quad j=\{1,2, \ldots, m-1\}
\end{gathered}
$$

The earliest times of all sectors in the first activity $(j=1)$ are determined by equations (26) and (27).

$$
\begin{gathered}
t_{1,1}^{w r}=0, \quad t_{i, 1}^{w r}=t_{i-1,1}^{w z}+S_{1, j}^{S} \\
t_{i, 1}^{w r}=\sum_{k=1}^{i-1} t_{k, 1}+S_{1, k}^{S}, \quad i=\{1,2, \ldots, n-1\}
\end{gathered}
$$

The couplings for the first activity are equal to the organizational couplings and don't 
affect the first sector. The couplings for the first sector are zero (as in TCM II) and don't affect the first activity.

$$
\begin{gathered}
S_{1, j}^{S W}=S_{i, 1}^{S} \\
S_{i, 1}^{F W}=0
\end{gathered}
$$

The earliest times of starting and finishing the work are determined by equations (30) and (31).

$$
t_{i, j}^{w r}=\max \left\{t_{i-1, j}^{w z}+s_{i, j}^{S}, t_{i, j-1}^{w z}\right\}
$$

The earliest times of finishing the work $(i, j)$ is determined by adding the earliest times of the starting work and it's duration.

$$
t_{i, j}^{w z}=t_{i, j}^{w r}+t_{i, j}
$$

In order to determinate other earliest times of starting the work $(i, j)$ the following couplings needs to be considered:

- The preceding work in activity modified by organization couplings $(i-1, j)$ - the continuity of activities

- The preceding work in sector $(\mathrm{i}, \mathrm{j}-1)$ - the continuity of work in sectors

The couplings between activities for the earliest times are determined by difference between earliest times of starting and finishing of two adjacent works in one activity modified by organization couplings.

$$
\begin{gathered}
S_{i, j}^{S W}=t_{i, j}^{w r}-t_{i-1, j}^{w z}+S_{i, j}^{S} \\
S_{i, j}^{F W}=t_{i, j}^{w r}-t_{i, j-1}^{w z}
\end{gathered}
$$

The latest times are determined by an assumption that the earliest and latest times of finishing work in the latest activity in latest sector are equal.

$$
t_{n, m}^{p z}=t_{n, m}^{w z}
$$

The latest times of all activities on last sector $(\mathrm{i}=\mathrm{n})$ are determined by equations ( 35$)$ and (36).

$$
\begin{gathered}
t_{n, j}^{p z}=t_{n, j+1}^{p r} \\
t_{n, j}^{p z}=t_{n, m}^{p z}-\sum_{k=j+1}^{m} t_{n, k}, \quad j=\{1,2, \ldots, m-1\}
\end{gathered}
$$

The latest times of all sectors in the last activity $(\mathrm{j}=\mathrm{m})$ are determined by equations (37) and (38).

$$
\begin{gathered}
t_{i, m}^{p z}=t_{i+1, m}^{p r}-S_{i, m}^{S} \\
t_{i, m}^{p z}=t_{n, m}^{p z}-\sum_{k=i+1}^{n} t_{k, m}-S_{k, m}^{S}, \quad i=\{1,2, \ldots, m-1\}
\end{gathered}
$$

Couplings for the last activity are equal to the organizational couplings and don't affect the last sector. Couplings for the last sector are zero (as in TCM II) and don't affect the last activity.

$$
\begin{gathered}
S_{i, m}^{S P}=S_{i, m}^{S} \\
S_{n, j}^{F P}=0
\end{gathered}
$$

The latest times of finishing and starting the work are determined by equations (41) and (42). 


$$
t_{i, j}^{p z}=\max \left\{t_{i-1, j}^{p r}-s_{i, j}^{S}, t_{i, j-1}^{p r}\right\}
$$

The latest times of starting the work $(i, j)$ is determined by subtracting the latest times of the finishing work and it's duration.

$$
t_{i, j}^{p r}=t_{i, j}^{p z}-t_{i, j}
$$

In order to determinate other latest times of finishing the work $(i, j)$ the following couplings needs to be considered:

- the following work in activity $(i+1, j)$ modified by organization couplings - the continuity of activities

- the following work in sector $(i, j+1)$ - the continuity of work in sectors

\section{Summary}

The article presents a modification one of the Time Coupling Methods. The one presented is to add more flexibility to scheduling problems regarding to organizational constrains. The presented procedure allow to make a sequence in which to carry out works, taking into account couplings between means of realization and couplings between work sectors. TCM is also open to optimize scheduling jobs using B\&B or metaheuristic algorithms.

The work was created as part of an international grant for a research project PWr -RUDN 2017: $\mathrm{O} / 0180 / 524 / 2017$

\section{References}

1. Afanasev V.A., Afanasev A.V. Stream scheduling of works in civil engineering. St. Petersburg (in Russian). 2000.

2. Arditi D., Tokdemir O.B. \& Suh K. Scheduling system for repetitive unit construction using line-of-balance technology. Scheduling system for repetitive unit construction using line-of-balance technology. Department of Civil and Architectural Engineering, Illinois Institute of Technology, Department of Civil Engineering, Honan University, Honam, South Korea. 433-446. 2001.

3. Faghihi S. V., Nejat A., Reinschmidt K., Kang J.H. Automation in construction scheduling: a review of the literature. International Journal of Advanced Manufacturing Technology 81(9), 2015.

4. Goldratt E.M. Critical Chain. Great Barrington, MA: The North River Press. 1997.

5. Hamerlink D. J., Rowings J. E. Linear scheduling model: Development of controlling activity path. Journal of Construction Engineering and Management, 124(4), 266-268. 1998.

6. Harris R. B. and Ioannou P.G. Scheduling Projects with Repeating Activities. Journal of Construction Engineering and Management. ASCE, Vol. 124, NO. 4, pp.269-278. 1998.

7. Hegazy T. Optimization of construction time -cost trade-off analisys using genetic algorithms. Canadian Journal of Civil Engineering, 26: 685-697. 1999.

8. Hejducki Z. Sprzężenia czasowe $w$ metodach organizacji złożonych procesów budowlanych. Monografie, WPWr. 2000.

9. Hejducki Z., Rogalska M. Metody sprzężeń czasowych TCM. Przegląd budowlany 2/2005, 38-45. 2005.

10. Hejducki Z., Rogalska M. Time coupling methods. Construction scheduling and time/cost optimization. Oficyna Wydawnicza Politechniki Wrocławskiej. 2011.

11. Hejducki Z., Rogalska M. Harmonogramowanie procesów budowlanych metodami sprzężén czasowych. Monografie, Politechnika Lubelska. 2017. 
12. Johnston D.W. Linear scheduling methods for highway construction. J. Constr. Div., ASCE, 107(C02), 247-261. 1981.

13. Lucko G. Flexible Modeling of Linear Schedules for Integrated Mathematical Analysis. Proceedings of the 2007 39th Winter Simulation Conference, eds. Henderson, S. G., Biller, B., Hsieh, M.-H., Shortle, J., Tew, J. D., Barton, R. R., Washington, District of Columbia, December 9-12, 2007, Institute of Electrical and Electronics Engineers, Piscataway, New Jersey, 2159-2167. 2007.

14. Lucko G. Productivity Scheduling Method Compared to Linear and Repetitive Project Scheduling Methods. Journal of Construction Engineering and Management 134(9): 711-720. 2008.

15. Lucko G. Productivity Scheduling Method: Linear Schedule Analysis with Singularity Functions. Journal of Construction Engineering and Management 135(4): 246-253. 2009.

16. Marcinkowski R. Metody rozdzialu zasobów realizatora $w$ działalności inżynieryjno budowlanej. Warszawa, WAT. 2002.

17. Mrozowicz J. Methods of organizing construction activities taking into account time couplings. (in Polish), Wrocław University of Technology Publishing House. 1997.

18. Połoński M. Application of the work breakdown structure in determining cost buffers in construction schedules. Archives of Civil Engineering. Volume LXI, Issue 1/2015, Pages 147-161, DOI: 10.1515/ace-2015-0010. 2015.

19. Rogalska M., Bożejko W., Hejducki Z. Time/cost optimization using hybrid evolutionary algorithm in construction project scheduling. Automation in Construction 18, 24-31. 2008.

20. Turkan Y., Bosche F., Haas C. T., Haas R. Automated progress tracking using 4D schedule and $3 D$ sensing technologies. Automation in Construction Vol.22, pp. 414421, 2012. 